\title{
Approximately dual Gabor frames and almost perfect reconstruction based on a class of window functions
}

\author{
Christensen, Ole; Janssen, Augustus J.E.M.; Kim, Hong Oh; Kim, Rae Young
}

Published in:

Advances in Computational Mathematics

Link to article, DOI:

10.1007/s10444-018-9595-7

Publication date:

2018

Document Version

Peer reviewed version

Link back to DTU Orbit

Citation (APA):

Christensen, O., Janssen, A. J. E. M., Kim, H. O., \& Kim, R. Y. (2018). Approximately dual Gabor frames and almost perfect reconstruction based on a class of window functions. Advances in Computational Mathematics, 44(5), 1519-1535. https://doi.org/10.1007/s10444-018-9595-7

\section{General rights}

Copyright and moral rights for the publications made accessible in the public portal are retained by the authors and/or other copyright owners and it is a condition of accessing publications that users recognise and abide by the legal requirements associated with these rights.

- Users may download and print one copy of any publication from the public portal for the purpose of private study or research.

- You may not further distribute the material or use it for any profit-making activity or commercial gain

- You may freely distribute the URL identifying the publication in the public portal 


\title{
Approximately dual Gabor frames and almost perfect reconstruction based on a class of window functions
}

\author{
O. Christensen - A. J. E. M. Janssen • \\ H. O. Kim • R. Y. Kim
}

Received: date / Accepted: date

\begin{abstract}
It is a well-known problem in Gabor analysis how to construct explicitly given dual frames associated with a given frame. In this paper we will consider a class of window functions for which approximately dual windows can be calculated explicitly. The method makes it possible to get arbitrarily close to perfect reconstruction by allowing the modulation parameter to vary. Explicit estimates for the deviation from perfect reconstruction are provided for some of the standard functions in Gabor analysis, e.g., the Gaussian and the two-sided exponential function.
\end{abstract}

Keywords Frames · approximately dual frames · almost perfect reconstruction $\cdot$ Gaussian $\cdot$ two-sided exponential

Mathematics Subject Classification (2000) $42 \mathrm{C} 40 \cdot 42 \mathrm{C} 15$

This research was supported by Basic Science Research Program through the National Research Foundation of Korea(NRF) funded by the Ministry of Education(2016R1D1A1B02009954).

O. Christensen

Department of Applied Mathematics and Computer Science, Technical University of Denmark, Building 303, 2800 Lyngby, Denmark E-mail: ochr@dtu.dk

A. J. E. M. Janssen

Department of Mathematics and Computer Science, Technische Universiteit Eindhoven, 5600 Eindhoven, The Netherlands E-mail: a.j.e.m.janssen@tue.nl

H. O. Kim

Department of Mathematical Sciences, UNIST, 50 UNIST-gil, Ulsan 44919, Republic of Korea

E-mail: hkim2031@unist.ac.kr

R. Y. Kim

Department of Mathematics, Yeungnam University, 280 Daehak-Ro, Gyeongsan, Gyeongbuk 38541, Republic of Korea

Tel.: +82-53-810-2319 Fax: +82-53-810-4614 E-mail: rykim@ynu.ac.kr 


\section{Introduction}

Gabor analysis is typically based on a choice of a window function $\varphi \in L^{2}(\mathbb{R})$ and suitable parameters $a, b>0$ such that the system $\left\{e^{2 \pi i m b x} \varphi(x-n a)\right\}_{m, n \in \mathbb{Z}}$ forms a frame for $L^{2}(\mathbb{R})$. A classical problem is that most applications need information about the dual window, which might be complicated to calculate explicitly. The purpose of this paper is to obtain explicit constructions of approximately dual windows for a class of windows $\mathcal{V} \subset L^{2}(\mathbb{R})$ that cover most of the classical choices in Gabor analysis. In contrast to the standard Gabor theory, we will allow the modulation parameter $b$ to change: indeed, we will prove that we can get arbitrarily close to perfect reconstruction for any $\varphi \in \mathcal{V}$ using a sufficiently small modulation parameter and the constructed approximately dual windows. Explicit estimates for the deviation from perfect reconstruction are provided for some of the standard functions in Gabor analysis, e.g., the Gaussian and the two-sided exponential function. Note that, in contrast to the approach in [3], the translation parameter will be kept fixed.

In the rest of this section we will set the stage by introducing the relevant class of window functions and collecting the necessary background information about frames and Gabor systems. In particular, a crucial ingredient in our method is to provide estimates for certain Bessel bounds; this is the topic of Section 2. We will indeed provide two estimates of the Bessel bounds: one that is valid for all functions $\varphi \in \mathcal{V}$, and a sharper estimate for convex functions. The construction of approximately dual windows for $g \in \mathcal{V}$ is given in Section 3.

A sequence $\left\{f_{k}\right\}_{k \in I}$ in a separable Hilbert space $\mathcal{H}$ is called a frame if there exist constants $A, B>0$ such that

$$
A\|f\|^{2} \leq \sum_{k \in I}\left|\left\langle f, f_{k}\right\rangle\right|^{2} \leq B\|f\|^{2}, \forall f \in \mathcal{H} .
$$

A sequence $\left\{f_{k}\right\}_{k \in I}$ satisfying at least the upper condition in (1.1) is called a Bessel sequence with Bessel bound B. It is well-known (see, e.g., [2]) that $\left\{f_{k}\right\}_{k \in I}$ is a Bessel sequence with bound $B$ if and only if

$$
\left\|\sum c_{k} f_{k}\right\|^{2} \leq B \sum\left|c_{k}\right|^{2}
$$

for all finite scalar sequences $\left\{c_{k}\right\}$.

Frames lead to unconditionally convergent series expansions of the elements in the underlying Hilbert space. Indeed, if $\left\{f_{k}\right\}_{k \in I}$ is a frame for $\mathcal{H}$, then there exists a frame $\left\{g_{k}\right\}_{k \in I}$ such that

$$
f=\sum_{k \in I}\left\langle f, g_{k}\right\rangle f_{k}=\sum_{k \in I}\left\langle f, f_{k}\right\rangle g_{k}, \forall f \in \mathcal{H} .
$$

A frame $\left\{g_{k}\right\}_{k \in I}$ that satisfies (1.2) is called a dual frame of $\left\{f_{k}\right\}_{k \in I}$; and (1.2) is expressed by saying that the frames $\left\{f_{k}\right\}_{k \in I}$ and $\left\{g_{k}\right\}_{k \in I}$ lead to perfect reconstruction. 
A weaker concept was introduced in [4]. We say that two Bessel sequences $\left\{f_{k}\right\}_{k \in I}$ and $\left\{g_{k}\right\}_{k \in I}$ form approximately dual frames if there exists a constant $\mu<1$ such that

$$
\left\|f-\sum_{k \in I}\left\langle f, f_{k}\right\rangle g_{k}\right\| \leq \mu\|f\|, \forall f \in \mathcal{H} .
$$

The rationale behind approximately dual frames is that all kinds of implementations involve certain imprecisions; thus, as long as we can control the deviation from perfect reconstruction measured by the parameter $\mu$ in (1.3), approximately dual frames can in practice be as good as exact dual frames. We refer to the papers $[1,5-7]$ for explicit constructions and applications of this concept. We will use the following elementary result from [3] to measure the deviation from perfect reconstruction.

Lemma 1 Let $\left\{f_{k}\right\}_{k \in I}$ be a frame with bounds $A, B$ and let $\left\{g_{k}\right\}_{k \in I}$ be a dual frame of $\left\{f_{k}\right\}_{k \in I}$ with upper frame bound $B_{g}$. If $\left\{\tilde{f}_{k}\right\}_{k \in I}$ is a sequence in $\mathcal{H}$ and

$$
\left\|\sum_{k \in I} c_{k}\left(f_{k}-\widetilde{f}_{k}\right)\right\| \leq \mu\left(\sum_{k \in I}\left|c_{k}\right|^{2}\right)^{1 / 2}
$$

for some $\mu \geq 0$ and all finite sequences $\left\{c_{k}\right\}_{k \in I}$, then

$$
\left\|f-\sum_{k \in I}\left\langle f, \widetilde{f}_{k}\right\rangle g_{k}\right\| \leq \mu \sqrt{B_{g}}\|f\|, \forall f \in \mathcal{H} .
$$

In particular $\left\{g_{k}\right\}_{k \in I}$ and $\left\{\widetilde{f_{k}}\right\}_{k \in I}$ are approximately dual frames if $\mu \sqrt{B_{g}}<1$.

For any parameters $a, b \in \mathbb{R}$, define the translation operator $T_{a}$ and modulation operators $E_{b}$ acting on $L^{2}(\mathbb{R})$ by

$$
T_{a} f(x)=f(x-a), E_{b} f(x)=e^{2 \pi i b x} f(x), x \in \mathbb{R} .
$$

Given a function $\varphi \in L^{2}(\mathbb{R})$ and parameters $a, b>0$, the associated Gabor system is the collection of functions $\left\{E_{m b} T_{n a} \varphi\right\}_{m, n \in \mathbb{Z}}$. The function $\varphi$ is called the window function.

Throughout this paper we will consider the following class of windows, which contains most of the standard choices in Gabor analysis.

Definition 1 Let $\mathcal{V} \subset L^{2}(\mathbb{R})$ denote the set consisting of the real-valued continuous functions $\varphi \in L^{2}(\mathbb{R})$ for which

(i) $\varphi$ is even and positive on $\mathbb{R}$;

(ii) $\varphi$ is decreasing on $[0, \infty[$;

(iii) $|\varphi(x)| \leq \frac{C}{1+|x|^{1+\sigma}}$ for some $C>0$ and $\sigma>0$.

For a fixed function $\varphi \in \mathcal{V}$ and any $N>0$, define the function $\varphi_{N} \in C_{c}(\mathbb{R})$ by

The name $\{$ lcal $V\}$ is
good because we don't
do wavelet analysis. I
will explain this in thecover
letter.

$$
\varphi_{N}(x):=(\varphi(x)-\varphi(N)) \chi_{[-N, N]}(x) .
$$


The function $\varphi_{N}$ in (1.6) can be considered to be a truncated version of $\varphi$, and will play a central role throughout this paper. Note that $N$ can be an arbitrary positive number in Definition 1; in Section 3 we will consider a sequence of approximately dual windows indexed by $N$, so in that case we only consider positive integer-values of $N$.

In the analysis of a given function $\varphi \in \mathcal{V}$ and its truncations $\varphi_{N}$, we will need the functions $Q_{N}:(0, \infty) \rightarrow \mathbb{R}$ defined by

$$
Q_{N}(x):=(\lfloor 2 x N\rfloor+1) \varphi(N)+2 \sum_{k=0}^{\infty} \varphi\left(N+\frac{k}{x}\right) .
$$

\section{Frame properties of functions $\varphi \in \mathcal{V}$ and $\varphi_{N}$}

In this section we will discuss the relationship between the frame properties of functions $\varphi \in \mathcal{V}$ and the associated truncations $\varphi_{N}$ in (1.6). The results will be based on Proposition 2, where we estimate the Bessel bound for the functions $\varphi-\varphi_{N}$. We will need the following estimate of the function $Q_{N}$ defined in $(1.7)$.

Lemma 2 Let $N>0$. For $\varphi \in \mathcal{V}$ and $C, \sigma>0$ as in Definition 1,

$$
Q_{N}(x) \leq \frac{C}{N^{1+\sigma}}\left(\lfloor 2 x N\rfloor+1+2\left(1+\frac{x N}{\sigma}\right)\right) .
$$

Proof. The decay condition (iii) in Definition 1 implies that

$$
\begin{aligned}
\sum_{k=0}^{\infty} \varphi\left(N+\frac{k}{x}\right) & \leq \sum_{k=0}^{\infty} \frac{C}{\left(N+\frac{k}{x}\right)^{1+\sigma}} \\
& \leq \frac{C}{N^{1+\sigma}}+\int_{0}^{\infty} \frac{C}{\left(N+\frac{t}{x}\right)^{1+\sigma}} d t=\frac{C}{N^{1+\sigma}}\left(1+\frac{x N}{\sigma}\right) .
\end{aligned}
$$

Thus

$$
\begin{aligned}
Q_{N}(x) & \leq(\lfloor 2 x N\rfloor+1) \frac{C}{N^{1+\sigma}}+\frac{2 C}{N^{1+\sigma}}\left(1+\frac{x N}{\sigma}\right) \\
& =\frac{C}{N^{1+\sigma}}\left(\lfloor 2 x N\rfloor+1+2\left(1+\frac{x N}{\sigma}\right)\right) .
\end{aligned}
$$

Using Lemma 2 we can now provide a Bessel bound for the functions

$\varphi(x)-\varphi_{N}(x)=\min \{\varphi(N), \varphi(x)\}=\varphi(x) \chi_{[-N, N]^{c}}(x)+\varphi(N) \chi_{[-N, N]}(x), x \in \mathbb{R}$, whenever $\varphi \in \mathcal{V}$. We will use the fact that for any $g \in L^{2}(\mathbb{R})$, if

$$
B(g, a, b):=\frac{1}{b} \sup _{x \in[0, a]} \sum_{k \in \mathbb{Z}}\left|\sum_{n \in \mathbb{Z}} g(x-n a) \overline{g(x-n a-k / b)}\right|<\infty,
$$


then $\left\{E_{m b} T_{n a} g\right\}_{m, n \in \mathbb{Z}}$ is a Bessel sequence with Bessel bound $B(g, a, b)$; see, e.g., [2], Theorem 11.4.2.

Proposition 2 Let $\varphi \in \mathcal{V}$, and let $a, b>0$ be given. Given $N>0$, define the function $Q_{N}$ by (1.7). Then the following hold:

(i) The Bessel bound $B\left(\varphi-\varphi_{N}, a, b\right)$ can be estimated by

$$
B\left(\varphi-\varphi_{N}, a, b\right) \leq \frac{1}{b} Q_{N}(b) Q_{N}\left(\frac{1}{a}\right) .
$$

(ii) If $b \leq \frac{1}{2 N}$, then

$$
B\left(\varphi-\varphi_{N}, a, b\right) \leq \frac{1}{b} Q_{N}(b) Q_{N}\left(\frac{1}{a}\right) \leq \frac{C^{2}}{b N^{1+2 \sigma}}\left(4+\frac{1}{\sigma}\right)\left(\frac{2}{a}\left(1+\frac{1}{\sigma}\right)+\frac{3}{N}\right) .
$$

Proof. For notational convenience, let $q_{N}(x):=\varphi(x)-\varphi_{N}(x)$. We note that

$$
B\left(q_{N}, a, b\right) \leq \frac{1}{b}\left(\sup _{x \in[0, a], n \in \mathbb{Z}} \sum_{k \in \mathbb{Z}}\left|q_{N}(x-n a-k / b)\right|\right)\left(\sup _{x \in[0, a]} \sum_{n \in \mathbb{Z}}\left|q_{N}(x-n a)\right|\right)
$$

and

$$
q_{N}(x)=\left\{\begin{array}{l}
\varphi(N), x \in[-N, N] \\
\varphi(x), x \in[-N, N]^{c} .
\end{array}\right.
$$

Estimate of $\sum_{k \in \mathbb{Z}}\left|q_{N}(x-n a-k / b)\right|:$ Fix $x \in[0, a]$ and $n \in \mathbb{Z}$; then $x-n a-k / b$ hits $[-N, N]$ for at most $\lfloor 2 b N\rfloor+1$ values of $k$, and thus yields a maximal contribution $(\lfloor 2 b N\rfloor+1) \varphi(N)$ by (2.4). By (2.4) again, the contribution from $x-n a-k / b$ hitting the interval $[N, \infty[$ is at most

$$
\sum_{k=0}^{\infty}\left|q_{N}\left(N+\frac{k}{b}\right)\right|=\sum_{k=0}^{\infty} \varphi\left(N+\frac{k}{b}\right),
$$

which converges by (iii) in Definition 1. Including also the contribution from ] $-\infty,-N]$ leads to

$$
\sum_{k \in \mathbb{Z}}\left|q_{N}(x-n a-k / b)\right| \leq(\lfloor 2 b N\rfloor+1) \varphi(N)+2 \sum_{k=0}^{\infty} \varphi\left(N+\frac{k}{b}\right)=Q_{N}(b) .
$$

Estimate of $\sum_{n \in \mathbb{Z}}\left|q_{N}(x-n a)\right|$ : Applying the above result with $b=1 / a$ yields that

$$
\sum_{n \in \mathbb{Z}}\left|q_{N}(x-n a)\right| \leq Q_{N}\left(\frac{1}{a}\right)=\left(\left\lfloor\frac{2 N}{a}\right\rfloor+1\right) \varphi(N)+2 \sum_{k=0}^{\infty} \varphi(N+a k) .
$$

Hence (2.3) together with (2.5) and (2.6) implies that the Bessel bound of $\left\{E_{m b} T_{n a} q_{N}\right\}_{m, n \in \mathbb{Z}}$ can be estimated as stated in (2.2). 
For the proof of (ii), applying (2.1) with $b \leq \frac{1}{2 N}$ yields that

$$
\begin{aligned}
Q_{N}(b) & \leq \frac{C}{N^{1+\sigma}}\left(\lfloor 2 b N\rfloor+1+2\left(1+\frac{b N}{\sigma}\right)\right) \\
& \leq \frac{C}{N^{1+\sigma}}\left(2+2\left(1+\frac{1}{2 \sigma}\right)\right)=\frac{C}{N^{1+\sigma}}\left(4+\frac{1}{\sigma}\right) .
\end{aligned}
$$

Also,

$$
\begin{aligned}
Q_{N}\left(\frac{1}{a}\right) & \leq \frac{C}{N^{1+\sigma}}\left(\left\lfloor\frac{2 N}{a}\right\rfloor+1+2\left(1+\frac{N}{a \sigma}\right)\right) \\
& \leq \frac{C}{N^{1+\sigma}}\left(\frac{2 N}{a}+1+2\left(1+\frac{N}{a \sigma}\right)\right)=\frac{C}{N^{\sigma}}\left(\frac{2}{a}\left(1+\frac{1}{\sigma}\right)+\frac{3}{N}\right) .
\end{aligned}
$$

The result in (i) now immediately leads to (ii).

Proposition 2 implies that if $\left\{E_{m b} T_{n a} \varphi\right\}_{m, n \in \mathbb{Z}}$ is a frame and $\varphi \in \mathcal{V}$, then also the truncated function $\varphi_{N}$ in (1.6) generates a frame whenever $N>0$ is sufficiently large. This generalizes a result from [3], dealing with the Gaussian.

Corollary 3 Let $a, b>0$ and $\varphi \in \mathcal{V}$ be given, and assume that $\left\{E_{m b} T_{n a} \varphi\right\}_{m, n \in \mathbb{Z}}$ is a frame for $L^{2}(\mathbb{R})$. Then for $N>0$ sufficiently large the Gabor system $\left\{E_{m b} T_{n a} \varphi_{N}\right\}_{m, n \in \mathbb{Z}}$ is a frame for $L^{2}(\mathbb{R})$.

Proof. Fixing $\varphi \in \mathcal{V}$ and $a, b>0$, Proposition 2 combined with Lemma 2 shows that the Bessel bound $B\left(\varphi-\varphi_{N}, a, b\right)$ tends to zero as $N \rightarrow \infty$. Thus, denoting a lower frame bound for $\left\{E_{m b} T_{n a} \varphi\right\}_{m, n \in \mathbb{Z}}$ by $A$, we have

$$
B\left(\varphi-\varphi_{N}, a, b\right)<A
$$

now displayed formula

for $N$ sufficiently large. The result now follows from standard results in frame perturbation theory (see Corollary 22.1.5 in [2]).

It is well-known that a continuous function with compact support cannot generate a Gabor Riesz basis (see, e.g., Proposition 13.2.4 in [2]). Since the perturbation condition used in the proof of Corollary 3 preserves the Riesz basis property, the result implies that no function $\varphi \in \mathcal{V}$ can generate a Riesz basis.

Note that the proof of Corollary 3 shows how large $N$ should be chosen in order to guarantee that $\left\{E_{m b} T_{n a} \varphi_{N}\right\}_{m, n \in \mathbb{Z}}$ is a frame; we just need to choose $N$ such that (2.7) holds. In order to apply this result it is crucial to have good estimates for the Bessel bound $B\left(\varphi-\varphi_{N}, a, b\right)$. In the following concrete example we will see that based on the estimates in Proposition 2, the frame property of $\left\{E_{m b} T_{n a} \varphi_{N}\right\}_{m, n \in \mathbb{Z}}$ is guaranteed for $N=3 / 4$, but not for $N=1 / \sqrt{2}$. This motivates the subsequent Proposition 5 where we obtain a sharper estimate for the Bessel bound $B\left(\varphi-\varphi_{N}, a, b\right)$ under the aditional condition that the function $\varphi$ is convex. Then, in Example 6 we return to the same concrete example and show that $\left\{E_{m b} T_{n a} \varphi_{N}\right\}_{m, n \in \mathbb{Z}}$ actually is a frame for $N=1 / \sqrt{2}$. 
Example 4 We consider

$$
\varphi(x)=2^{1 / 4} e^{-\pi x^{2}}, x \in \mathbb{R},
$$

and $a=b=1 / \sqrt{2}$. According to (6.7-8) in [8], the optimal frame bounds of $\left\{E_{m b} T_{n a} \varphi\right\}_{m, n \in \mathbb{Z}}$ are given by

$$
A=2 \sum_{k, \ell \in \mathbb{Z}}(-1)^{k+\ell} e^{-\pi\left(k^{2}+\ell^{2}\right)}=2\left(\sum_{k=-\infty}^{\infty}(-1)^{k} e^{-\pi k^{2}}\right)^{2}
$$

and

$$
B=2 \sum_{k, \ell \in \mathbb{Z}} e^{-\pi\left(k^{2}+\ell^{2}\right)}=2\left(\sum_{k=-\infty}^{\infty} e^{-\pi k^{2}}\right)^{2},
$$

respectively. By a numerical computation, $A=1.669253683, B=2.360681198$.

Furthermore, using (2.2) and (1.7),

$$
\begin{aligned}
B\left(\varphi-\varphi_{N}, \frac{1}{\sqrt{2}}, \frac{1}{\sqrt{2}}\right) \leq & \sqrt{2} Q_{N}\left(\frac{1}{\sqrt{2}}\right) Q_{N}(\sqrt{2}) \\
\leq & 2\left((\lfloor N \sqrt{2}\rfloor+1) e^{-\pi N^{2}}+2 \sum_{k=0}^{\infty} e^{-\pi(N+k \sqrt{2})^{2}}\right) \\
& \times\left((\lfloor 2 N \sqrt{2}\rfloor+1) e^{-\pi N^{2}}+2 \sum_{k=0}^{\infty} e^{-\pi\left(N+\frac{k \sqrt{2}}{2}\right)^{2}}\right) .
\end{aligned}
$$

A numerical inspection shows that for $N=3 / 4$ this estimate of $B\left(\varphi-\varphi_{N}, \frac{1}{\sqrt{2}}, \frac{1}{\sqrt{2}}\right)$ is indeed smaller than the lower frame bound $A$ of $\left\{E_{m b} T_{n a} \varphi\right\}_{m, n \in \mathbb{Z}}$; thus $\left\{E_{m b} T_{n a} \varphi_{N}\right\}_{m, n \in \mathbb{Z}}$ is a frame for $N=3 / 4$. However, for $N=1 / \sqrt{2}$ the estimate of $B\left(\varphi-\varphi_{N}, \frac{1}{\sqrt{2}}, \frac{1}{\sqrt{2}}\right)$ exceeds the lower frame bound $A$ of $\left\{E_{m b} T_{n a} \varphi\right\}_{m, n \in \mathbb{Z}}$; thus, the proof of Corollary 3 does not guarantee that $\left\{E_{m b} T_{n a} \varphi_{N}\right\}_{m, n \in \mathbb{Z}}$ is a frame for $N=1 / \sqrt{2}$.

For windows $\varphi \in \mathcal{V}$ that are convex on $[N, \infty[$ we will now derive the following improvement of Proposition 2.

Proposition 5 Assume that $\varphi \in \mathcal{V}$ is convex on $[N, \infty[$ for some $N>0$. Then

$$
B\left(q_{N}, a, b\right) \leq \frac{1}{b}\left(\sum_{k=-\infty}^{\infty} q_{N}\left(N-\frac{k}{b}\right)\right)\left(\sum_{n=-\infty}^{\infty} q_{N}(N-n a)\right)
$$


Proof. By (2.3) and non-negativity of the function $q_{N}(x)=\min \{\varphi(x), \varphi(N)\}$, we have that

$$
B\left(q_{N}, a, b\right) \leq \frac{1}{b} \sup _{x \in[0,1 / b]}\left(\sum_{k=-\infty}^{\infty}\left|q_{N}(x-k / b)\right|\right) \sup _{x \in[0, a]}\left(\sum_{n=\infty}^{\infty}\left|q_{N}(x-n a)\right|\right) .
$$

We will first show that, under the assumption that $\varphi$ is convex on $[N, \infty[$,

$$
\begin{aligned}
& \sup _{x \in[0, a]}\left(\sum_{n \in \mathbb{Z}} q_{N}(x-n a)\right)=\sum_{n=-\infty}^{\infty} q_{N}(N-n a) \\
& =\left(\left\lfloor\frac{2 N}{a}\right\rfloor+1\right) \varphi(N)+\sum_{n=1}^{\infty} \varphi(N+n a)+\sum_{n=1}^{\infty} \varphi\left(\left\lfloor\frac{2 N}{a}\right\rfloor a-N+n a\right) .
\end{aligned}
$$

To show this, let $x \in] N-a, N]$; furthermore, let $n(x)$ be the largest integer $n$ such that $x-n a \geq-N$, i.e., $n(x)=\lfloor(N+x) / a\rfloor$. We distinguish between the cases that $n(x)=\lfloor 2 N / a\rfloor$ and $n(x)=\lfloor 2 N / a\rfloor-1$.

First assume that $n(x)=\lfloor 2 N / a\rfloor$. Then

$$
N-a \leq\left\lfloor\frac{2 N}{a}\right\rfloor a-N \leq x \leq N,
$$

and

$$
\begin{aligned}
& \sum_{n=-\infty}^{\infty} q_{N}(x-n a)=\sum_{n=-\infty}^{-1} \varphi(x-n a)+\sum_{n=0}^{n(x)} \varphi(N)+\sum_{n=n(x)+1}^{\infty} \varphi(x-n a) \\
& =\left(\left\lfloor\frac{2 N}{a}\right\rfloor+1\right) \varphi(N)+\sum_{n=1}^{\infty} \varphi(x+n a)+\sum_{n=1}^{\infty} \varphi\left(x-\left\lfloor\frac{2 N}{a}\right\rfloor a-n a\right) \\
& =\left(\left\lfloor\frac{2 N}{a}\right\rfloor+1\right) \varphi(N)+\sum_{n=1}^{\infty}\left[\varphi(x+n a)+\varphi\left(\left\lfloor\frac{2 N}{a}\right\rfloor a-x+n a\right)\right],(2 .
\end{aligned}
$$

where it has been used that $\varphi$ is even. In particular this proves the second equality sign in (2.8) in the considered case; so we only need to prove that the supremum is attained for $x=N$. If $\frac{2 N}{a}$ is integer we have that $\left\lfloor\frac{2 N}{a}\right\rfloor=\frac{2 N}{a}$, in which case (2.9) shows that the only $x$-value that can be considered under the given conditions indeed is $x=N$. We will therefore now focus on the case where $\frac{2 N}{a}$ is not an integer. In this case $2 N-\left\lfloor\frac{2 N}{a}\right\rfloor a>0$. Now let $n=1,2, \cdots$. We have

$$
\left\lfloor\frac{2 N}{a}\right\rfloor a-N+n a \leq x+n a \leq N+n a,
$$

and

$$
\left\lfloor\frac{2 N}{a}\right\rfloor a-N+n a \leq\left\lfloor\frac{2 N}{a}\right\rfloor a-x+n a \leq N+n a .
$$


Hence, letting

$$
\lambda:=\frac{N+n a-(x+n a)}{N+n a-\left(\left\lfloor\frac{2 N}{a}\right\rfloor a-N+n a\right)}=\frac{N-x}{2 N-\left\lfloor\frac{2 N}{a}\right\rfloor a} \in[0,1],
$$

the convexity of $\varphi$ implies that

$$
\begin{aligned}
\varphi(x+n a) & =\varphi\left(\lambda\left(\left\lfloor\frac{2 N}{a}\right\rfloor a-N+n a\right)+(1-\lambda)(N+n a)\right) \\
& \leq \lambda \varphi\left(\left\lfloor\frac{2 N}{a}\right\rfloor a-N+n a\right)+(1-\lambda) \varphi(N+n a) .
\end{aligned}
$$

Similarly, letting

$$
\mu:=\frac{N+n a-\left(\left\lfloor\frac{2 N}{a}\right\rfloor a-x+n a\right)}{N+n a-\left(\left\lfloor\frac{2 N}{a}\right\rfloor a-N+n a\right)}=\frac{N-\left\lfloor\frac{2 N}{a}\right\rfloor a+x}{2 N-\left\lfloor\frac{2 N}{a}\right\rfloor a} \in[0,1],
$$

we see that

$$
\begin{aligned}
\varphi\left(\left\lfloor\frac{2 N}{a}\right\rfloor a-x+n a\right) & =\varphi\left(\mu\left(\left\lfloor\frac{2 N}{a}\right\rfloor a-N+n a\right)+(1-\mu)(N+n a)\right) \\
& \leq \mu \varphi\left(\left\lfloor\frac{2 N}{a}\right\rfloor a-N+n a\right)+(1-\mu) \varphi(N+n a) .
\end{aligned}
$$

Observe that $\lambda+\mu=1$. Thus we get from (2.11) and (2.12) that

$$
\begin{aligned}
& \varphi(x+n a)+\varphi\left(\left\lfloor\frac{2 N}{a}\right\rfloor a-x+n a\right) \\
& \leq \lambda \varphi\left(\left\lfloor\frac{2 N}{a}\right\rfloor a-N+n a\right)+(1-\lambda) \varphi(N+n a) \\
& +(1-\lambda) \varphi\left(\left\lfloor\frac{2 N}{a}\right\rfloor a-N+n a\right)+\lambda \varphi(N+n a) \\
& =\varphi\left(\left\lfloor\frac{2 N}{a}\right\rfloor a-N+n a\right)+\varphi(N+n a) .
\end{aligned}
$$

Therefore, from (2.10),

$$
\begin{aligned}
\sum_{n=-\infty}^{\infty} q_{N}(x-n a) \leq & \left(\left\lfloor\frac{2 N}{a}\right\rfloor+1\right) \varphi(N) \\
& +\sum_{n=1}^{\infty}\left(\varphi(N+n a)+\varphi\left(\left\lfloor\frac{2 N}{a}\right\rfloor a-N+n a\right)\right) \\
= & \sum_{n=-\infty}^{\infty} q_{N}(N-n a) .
\end{aligned}
$$


This proves (2.8) in the case $n(x)=\lfloor 2 N / a\rfloor$, as desired. We now consider the case $n(x)=\lfloor 2 N / a\rfloor-1$. Then

$$
N-a<x \leq\left\lfloor\frac{2 N}{a}\right\rfloor a-N \leq N,
$$

and in particular, $\left\lfloor\frac{2 N}{a}\right\rfloor a-2 N+a>0$. With the interpretation that $\sum_{n=0}^{n(x)} \varphi(N)=$ 0 if $n(x)=-1$, we now have that

$$
\begin{aligned}
& \sum_{n=-\infty}^{\infty} q_{N}(x-n a)=\sum_{n=-\infty}^{-1} \varphi(x-n a)+\sum_{n=0}^{n(x)} \varphi(N)+\sum_{n=n(x)+1}^{\infty} \varphi(x-n a) \\
& =\left\lfloor\frac{2 N}{a}\right\rfloor \varphi(N)+\sum_{n=1}^{\infty} \varphi(x+n a)+\sum_{n=0}^{\infty} \varphi\left(x-\left\lfloor\frac{2 N}{a}\right\rfloor a-n a\right) \\
& =\left\lfloor\frac{2 N}{a}\right\rfloor \varphi(N)+\sum_{n=0}^{\infty}\left[\varphi(x+(n+1) a)+\varphi\left(\left\lfloor\frac{2 N}{a}\right\rfloor a-x+n a\right)\right] .
\end{aligned}
$$

We compare this to the right-hand side of (2.8), which we write as

$$
\left\lfloor\frac{2 N}{a}\right\rfloor \varphi(N)+\sum_{n=0}^{\infty}\left[\varphi(N+n a)+\varphi\left(\left\lfloor\frac{2 N}{a}\right\rfloor a-N+(n+1) a\right)\right] .
$$

Now for $n=0,1, \ldots$,

$$
N+n a<x+(n+1) a \leq\left\lfloor\frac{2 N}{a}\right\rfloor a-N+(n+1) a,
$$

and

$$
N+n a \leq\left\lfloor\frac{2 N}{a}\right\rfloor a-x+n a<\left\lfloor\frac{2 N}{a}\right\rfloor a-N+(n+1) a .
$$

Now, let

$$
\lambda:=\frac{\left\lfloor\frac{2 N}{a}\right\rfloor a-N-x}{\left\lfloor\frac{2 N}{a}\right\rfloor a-2 N+a}, \mu:=\frac{x-N+a}{\left\lfloor\frac{2 N}{a}\right\rfloor a-2 N+a} ;
$$

then $0 \leq \lambda, \mu \leq 1$ and $\lambda+\mu=1$. Hence, writing

$$
x+(n+1) a=\lambda(N+n a)+(1-\lambda)\left(\left\lfloor\frac{2 N}{a}\right\rfloor a-N+(n+1) a\right),
$$

and

$$
\left\lfloor\frac{2 N}{a}\right\rfloor a-x+n a=\mu(N+n a)+(1-\mu)\left(\left\lfloor\frac{2 N}{a}\right\rfloor a-N+(n+1) a\right),
$$

the convexity of $\varphi$ implies that

$$
\begin{aligned}
& \varphi(x+(n+1) a)+\varphi\left(\left\lfloor\frac{2 N}{a}\right\rfloor a-x+n a\right) \\
& \leq \varphi(N+n a)+\varphi\left(\left\lfloor\frac{2 N}{a}\right\rfloor a-N+(n+1) a\right) .
\end{aligned}
$$


Hence

$$
\sum_{n=-\infty}^{\infty} q_{N}(x-n a) \leq \sum_{n=-\infty}^{\infty} q_{N}(N-n a)
$$

and the proof of (2.8) is complete.

Now, to finalize the proof we observe that the convexity condition on $\varphi$ ensuring the validity of (2.8) does not involve $a$. Hence, using (2.8) once more with $1 / b$ replacing $a$, we get

$$
B\left(q_{N}, a, b\right) \leq \frac{1}{b}\left(\sum_{k=-\infty}^{\infty} q_{N}\left(N-\frac{k}{b}\right)\right)\left(\sum_{n=-\infty}^{\infty} q_{N}(N-n a)\right)
$$

as claimed.

We will now return to the function in Example 4 and show that the sharper estimates in Proposition 5 indeed improves the result.

Example 6 We consider again the function $\varphi$ in Example 4 and $a=b=$ $\sqrt{2} / 2$. We have that $\varphi^{\prime \prime}(x) \geq 0 \Leftrightarrow|x| \geq 1 / \sqrt{2 \pi}=0.398942280$. We take $N=1 / \sqrt{2 \pi}$, so that

$$
\left\lfloor\frac{2 N}{a}\right\rfloor=\left\lfloor\frac{2}{\sqrt{\pi}}\right\rfloor=1,\lfloor 2 N b\rfloor=\left\lfloor\frac{1}{\sqrt{\pi}}\right\rfloor=0 .
$$

Using (2.8), the right-hand side of (2.13) can be written as

$$
\begin{aligned}
& 2\left(2 e^{-\pi N^{2}}+\sum_{n=1}^{\infty} e^{-\pi\left(N+\frac{1}{2} n \sqrt{2}\right)^{2}}+\sum_{n=1}^{\infty} e^{-\pi\left(-N+\frac{1}{2}(n+1) \sqrt{2}\right)^{2}}\right) \\
& \times\left(e^{-\pi N^{2}}+\sum_{k=1}^{\infty} e^{-\pi(N+k \sqrt{2})^{2}}+\sum_{k=1}^{\infty} e^{-\pi(-N+k \sqrt{2})^{2}}\right) \\
& =1.645277057
\end{aligned}
$$

and this is just less than the lower frame bound $A$, which we found in Example 4. Thus $\left\{E_{m b} T_{n a} \varphi_{N}\right\}_{m, n \in \mathbb{Z}}$ is indeed a frame for $N=1 / \sqrt{2}$, a conclusion that we could not reach based on Proposition 2.

Plots of the functions $\varphi(x), \varphi_{3 / 4}(x)$ and $\varphi_{1 / \sqrt{2 \pi}}(x)$ are shown in Figure 1. Note that the length of the support of the function $\varphi_{1 / \sqrt{2 \pi}}$ is $2 / \sqrt{2 \pi}$, which just exceeds $a=\sqrt{2} / 2$. Thus Proposition 5 gives a sharp result for this case; indeed, $\left\{E_{m b} T_{n a} \varphi_{N}\right\}_{m, n \in \mathbb{Z}}$ ceases to be a Gabor frame when $2 N \leq a$.

We will now show that with particular knowledge of certain relations between the parameters $a, b>0$ and $N>0$ we can be more specific about the frame properties of the functions $\varphi_{N}$ for $\varphi \in \mathcal{V}$, even without assuming that $\left\{E_{m b} T_{n a} \varphi\right\}_{m, n \in \mathbb{Z}}$ is a frame. 


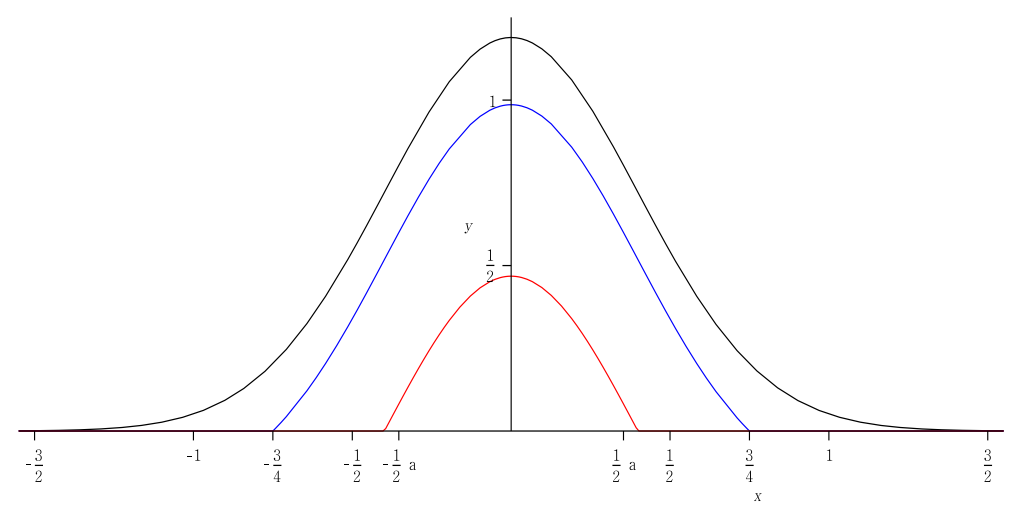

Fig. 1 The function $\varphi(x)=2^{1 / 4} e^{-\pi x^{2}}$ (black) and the function $\varphi_{N}$ for $N=3 / 4$ (blue; see Proposition 2) and for $N=1 / \sqrt{2 \pi}$ (red; see Proposition 5).

Proposition 7 Let $\varphi \in \mathcal{V}$. For $N>0$, consider the function $\varphi_{N}$ in (1.6) and let $\left.a \in] 0,2 N[, b \in] 0, \frac{1}{2 N}\right]$. Then $\left\{E_{m b} T_{n a} \varphi_{N}\right\}_{m, n \in \mathbb{N}}$ is a frame for $L^{2}(\mathbb{R})$ with lower frame bound $A\left(\varphi_{N}\right)=\frac{1}{b}\left|\varphi_{N}\left(\frac{a}{2}\right)\right|^{2}$, and the canonical dual window is given by

$$
\tilde{\varphi}_{N}(x)=\frac{b \varphi_{N}(x)}{\sum_{n \in \mathbb{Z}}\left|\varphi_{N}(x-n a)\right|^{2}}, x \in \mathbb{R} .
$$

Proof. We first show that

$$
b A\left(\varphi_{N}\right) \leq \sum_{n \in \mathbb{Z}}\left|\varphi_{N}(x-n a)\right|^{2} \leq b B\left(\varphi_{N}\right), x \in \mathbb{R},
$$

for some $A\left(\varphi_{N}\right), B\left(\varphi_{N}\right)>0$. Since $\varphi_{N}$ is bounded and compactly supported, the upper bound is clear. Now, since the function $\sum_{n \in \mathbb{Z}}\left|\varphi_{N}(x-n a)\right|^{2}$ is $a$ periodic, we have

$$
\inf _{x \in \mathbb{R}} \sum_{n \in \mathbb{Z}}\left|\varphi_{N}(x-n a)\right|^{2}=\inf _{x \in\left[-\frac{a}{2}, \frac{a}{2}\right]} \sum_{n \in \mathbb{Z}}\left|\varphi_{N}(x-n a)\right|^{2} \geq \inf _{x \in\left[-\frac{a}{2}, \frac{a}{2}\right]}\left|\varphi_{N}(x)\right|^{2} .
$$

Since $\varphi \in \mathcal{V}$ is even, it follows that

$$
\inf _{x \in \mathbb{R}} \sum_{n \in \mathbb{Z}}\left|\varphi_{N}(x-n a)\right|^{2} \geq\left|\varphi_{N}\left(\frac{a}{2}\right)\right|^{2}=b\left(\frac{1}{b}\left|\varphi_{N}\left(\frac{a}{2}\right)\right|^{2}\right)=: b A\left(\varphi_{N}\right)>0 .
$$

Hence $\varphi_{N}$ satisfies (2.15). The result now follows by [2, Corollary 11.4.5].

Using Proposition 7 we can now show that for a fixed $a>0$, any function $\varphi \in \mathcal{V}$ generates a Gabor frame for sufficiently small modulation parameters.

Corollary 8 Let $\varphi \in \mathcal{V}$ and $a>0$. Then $\left\{E_{m b} T_{n a} \varphi\right\}_{m, n \in \mathbb{Z}}$ is a frame for $L^{2}(\mathbb{R})$ whenever $b>0$ is sufficiently small. 
Proof. Proposition 2 (ii) shows that the Bessel bound $B\left(\varphi-\varphi_{N}, a, b\right)$ tends to zero as $N \rightarrow \infty$. On the other hand, by Proposition 7 the function $\varphi_{N}$ generates a Gabor frame $\left\{E_{m b} T_{n a} \varphi_{N}\right\}_{m, n \in \mathbb{Z}}$ for $N>a / 2$, with lower frame bound $A\left(\varphi_{N}\right)=\frac{1}{b}\left|\varphi_{N}\left(\frac{a}{2}\right)\right|^{2}$. Since $\varphi_{N}\left(\frac{a}{2}\right) \rightarrow g\left(\frac{a}{2}\right)>0$, as $N \rightarrow \infty$, the result now follows from standard results in frame perturbation theory (see Corollary 22.1.5 in [2]).

\section{Approximately dual frames for $\varphi \in \mathcal{V}$}

Even when a function $\varphi \in \mathcal{V}$ generates a frame $\left\{E_{m b} T_{n a} \varphi\right\}_{m, n \in \mathbb{Z}}$, information about its dual frames might not be easily available. Thus, we do not have immediate access to apply the perfect reconstruction formula associated with a pair of dual frames. The purpose of this section is to provide a method for obtaining almost perfect reconstruction by using the fact that explicit dual frames associated to the truncated windows $\varphi_{N}$ in (1.6) can be calculated for certain parameter values $a, b>0$, see Proposition 7 . Note that Theorem 9 below is formulated via a frame condition on the truncated function $\varphi_{N}$, not on the function $\varphi \in \mathcal{V}$. This formulation matches the actual application to various concrete functions that will be given in Corollary 11 .

Theorem 9 Let $a, b$ be given such that $a b \in] 0,1[$. Given a function $\varphi \in \mathcal{V}$ and $N \in \mathbb{N}$, assume that $\left\{E_{m b} T_{n a} \varphi_{N}\right\}_{m, n \in \mathbb{Z}}$ is a frame for $L^{2}(\mathbb{R})$ and let $\left\{E_{m b} T_{n a} \varphi_{N}^{\sharp}\right\}_{m, n \in \mathbb{Z}}$ denote a dual frame with upper bound $B\left(\varphi_{N}^{\sharp}\right)$. Then

$$
\left\|f-\sum_{m, n \in \mathbb{Z}}\left\langle f, E_{m b} T_{n a} \varphi\right\rangle E_{m b} T_{n a} \varphi_{N}^{\sharp}\right\| \leq \sqrt{\frac{1}{b} Q_{N}(b) Q_{N}\left(\frac{1}{a}\right) B\left(\varphi_{N}^{\sharp}\right)}\|f\|, \forall f \in L^{2}(\mathbb{R}) .
$$

Proof. We apply Lemma 1 to the dual frames

$$
\left\{f_{k}\right\}_{k \in I}:=\left\{E_{m b} T_{n a} \varphi_{N}\right\}_{m, n \in \mathbb{Z}},\left\{g_{k}\right\}_{k \in I}:=\left\{E_{m b} T_{n a} \varphi_{N}^{\sharp}\right\}_{m, n \in \mathbb{Z}} .
$$

Letting $\left\{\widetilde{f}_{k}\right\}_{k \in I}:=\left\{E_{m b} T_{n a} \varphi\right\}_{m, n \in \mathbb{Z}}$, the condition (1.4) is satisfied with $\mu^{2}=B\left(\varphi-\varphi_{N}, a, b\right)$. Thus, the factor $\mu \sqrt{B_{g}}$ in (1.5) corresponds precisely to $\left(B\left(\varphi-\varphi_{N}, a, b\right) B\left(\varphi_{N}^{\sharp}\right)\right)^{1 / 2}$. The result now follows by Lemma 1 and Proposition 2 .

Remark 10 Note that if $\varphi \in \mathcal{V}$ generates a Gabor frame $\left\{E_{m b} T_{n a} \varphi\right\}_{m, n \in \mathbb{Z}}$, then Corollary 3 shows that $\left\{E_{m b} T_{n a} \varphi_{N}\right\}_{m, n \in \mathbb{Z}}$ is a frame for $N \in \mathbb{N}$ sufficiently large; thus the condition in Theorem 9 is satisfied. In our applications of Theorem 9 we will take $\varphi_{N}^{\sharp}$ to be the canonical dual window associated with the window $\varphi_{N}$. In this case $\left\{E_{m b} T_{n a} \varphi_{N}^{\sharp}\right\}_{m, n \in \mathbb{Z}}$ has the upper bound $B\left(\varphi_{N}^{\sharp}\right)=A\left(\varphi_{N}\right)^{-1}$, where $A\left(\varphi_{N}\right)$ denotes a lower frame bound for the frame $\left\{E_{m b} T_{n a} \varphi_{N}\right\}_{m, n \in \mathbb{Z}}$. 
For a fixed function $\varphi \in \mathcal{V}$ and fixed parameters $a, b>0$ and $N \in \mathbb{N}$, Theorem 9 yields an explicit estimate of the deviation from perfect reconstruction that is obtained by doing analysis with the Gabor system $\left\{E_{m b} T_{n a} \varphi\right\}_{m, n \in \mathbb{Z}}$ and synthesis using a dual frame $\left\{E_{m b} T_{n a} \varphi_{N}^{\sharp}\right\}_{m, n \in \mathbb{Z}}$. We will now consider the particular case where the synthesis is done using the canonical dual frame $\left\{E_{m b} T_{n a} \widetilde{\varphi}_{N}\right\}_{m, n \in \mathbb{Z}}$ and show that by allowing the modulation parameter $b$ to vary we can get as close to perfect reconstruction as desired.

Corollary 11 Let $a \in] 0, \infty\left[\right.$ and let $\{b(N)\}_{N=1}^{\infty} \subset \mathbb{R}$ be a sequence such that $0<b(N) \leq \frac{1}{2 N}$. Given a function $\varphi \in \mathcal{V}$, consider the functions $\varphi_{N}$ in (1.6) and $\widetilde{\varphi}_{N}$ in (2.14) for any integer $N>a / 2$ and with $b=b(N)$. Then, with the constants $C, \sigma>0$ chosen as in Definition 1,

$$
\left\|f-\sum_{m, n \in \mathbb{Z}}\left\langle f, E_{m b(N)} T_{n a} \varphi\right\rangle E_{m b(N)} T_{n a} \tilde{\varphi}_{N}\right\| \leq \frac{C_{1}(N)}{N^{\frac{1}{2}+\sigma}}\|f\|, \forall f \in L^{2}(\mathbb{R}),
$$

where

$$
C_{1}(N):=\frac{2 C}{\varphi_{N}\left(\frac{a}{2}\right)} \sqrt{\left(4+\frac{1}{\sigma}\right)\left(\frac{2}{a}\left(1+\frac{1}{\sigma}\right)+\frac{3}{N}\right)} .
$$

Proof. Proposition 2 (ii) implies that

$$
\frac{1}{b(N)} Q_{N}(b(N)) Q_{N}\left(\frac{1}{a}\right) \leq \frac{C^{2}}{b(N) N^{1+2 \sigma}}\left(4+\frac{1}{\sigma}\right)\left(\frac{2}{a}\left(1+\frac{1}{\sigma}\right)+\frac{3}{N}\right) .
$$

Considering now $N \in \mathbb{N}$ such that $N>a / 2$ and using that $0<b(N) \leq$ $\frac{1}{2 N}$, Proposition 7 shows that $\left\{E_{m b(N)} T_{n a} \varphi_{N}\right\}_{m, n \in \mathbb{Z}}$ is a Gabor frame with the canonical dual frame $\left\{E_{m b(N)} T_{n a} \tilde{\varphi}_{N}\right\}_{m, n \in \mathbb{Z}}$. Furthermore, a lower frame bound is $A\left(\varphi_{N}\right)=\frac{\left|\varphi_{N}\left(\frac{a}{2}\right)\right|^{2}}{b(N)}$. This together with (3.3) implies that

$$
\sqrt{\frac{1}{b(N)} Q(b(N)) Q\left(\frac{1}{a}\right) \frac{1}{A\left(\varphi_{N}\right)}} \leq \frac{C_{1}(N)}{N^{\frac{1}{2}+\sigma}},
$$

where $C_{1}(N)$ is defined by (3.2). Now (3.1) is a consequence of Theorem 9 and Remark 10.

Note that Corollary 11 immediately applies to the "canonical functions" in $\mathcal{V}$, i.e.,

$$
\varphi(x)=\frac{1}{1+|x|^{1+\sigma}},
$$

for $\sigma>0$. In the case $\sigma=1$ this function corresponds (up to a scalar multiple of the variable) to the Fourier transform of the two-sided exponential function $g(x)=e^{-|x|}$, which is known to generate a Gabor frame for all parameter 
values $a, b>0$ with $a b<1$, see [9]. It follows that also the function $\varphi(x)=$ $\left(1+x^{2}\right)^{-1}$ generates a Gabor frame for all $a b<1$.

In the next result we improve the estimate in Corollary 11 for two standard functions, namely, the Gaussian and the two-sided exponential function. In order to get as close as desired to perfect reconstruction, the parameter $N$ has to be chosen sufficiently large; the result shows how $N$ depends on the chosen function $\varphi \in \mathcal{V}$.

Corollary 12 Let $a \in] 0, \infty\left[\right.$ and let $\{b(N)\}_{N=1}^{\infty} \subset \mathbb{R}$ be a sequence such that $0<b(N) \leq \frac{1}{2 N}$. Given a function $\varphi \in \mathcal{V}$, consider again the functions $\varphi_{N}$ in (1.6) and $\widetilde{\varphi}_{N}$ in (2.14) for any integer $N>a / 2$ and with $b=b(N)$.

(1) Consider $\varphi(x)=e^{-|x|}, x \in \mathbb{R}$. Then

$$
\left\|f-\sum_{m, n \in \mathbb{Z}}\left\langle f, E_{m b(N)} T_{n a} \varphi\right\rangle E_{m b(N)} T_{n a} \tilde{\varphi}_{N}\right\| \leq C_{2}(N) \sqrt{N} e^{-N}\|f\|, \forall f \in L^{2}(\mathbb{R}),
$$

where

$$
C_{2}(N):=\frac{e^{\frac{a}{2}}}{\left(1-e^{-N+\frac{a}{2}}\right)} \sqrt{\left(2+\frac{2}{1-e^{-2 N}}\right)\left(\frac{2}{a}+\frac{1}{N}\left(\frac{3-e^{-a}}{1-e^{-a}}\right)\right)} ;
$$

(2) Consider $\varphi(x)=e^{-x^{2}}, x \in \mathbb{R}$. Then

$$
\left\|f-\sum_{m, n \in \mathbb{Z}}\left\langle f, E_{m b(N)} T_{n a} \varphi\right\rangle E_{m b(N)} T_{n a} \tilde{\varphi}_{N}\right\| \leq C_{3}(N) \sqrt{N} e^{-N^{2}}\|f\|, \forall f \in L^{2}(\mathbb{R}),
$$

where

$$
C_{3}(N):=\frac{e^{\frac{a^{2}}{4}}}{\left(1-e^{-N^{2}+\frac{a^{2}}{4}}\right)} \sqrt{\left(2+\frac{2}{1-e^{-4 N^{2}}}\right)\left(\frac{2}{a}+\frac{1}{N}\left(\frac{3-e^{-2 N a}}{1-e^{-2 N a}}\right)\right)} .
$$

Proof. For (1), applying (1.7) and $b(N) \leq \frac{1}{2 N}$ yields that

$$
\begin{aligned}
Q_{N}(b(N)) & =(\lfloor 2 b(N) N\rfloor+1) \varphi(N)+2 \sum_{k=0}^{\infty} \varphi\left(N+\frac{k}{b(N)}\right) \\
& \leq 2 \varphi(N)+2 \sum_{k=0}^{\infty} \varphi(N+2 N k)=e^{-N}\left(2+\frac{2}{1-e^{-2 N}}\right) .
\end{aligned}
$$

Similarly,

$$
\begin{aligned}
Q_{N}\left(\frac{1}{a}\right) & =\left(\left\lfloor\frac{2 N}{a}\right\rfloor+1\right) \varphi(N)+2 \sum_{k=0}^{\infty} \varphi(N+a k) \\
& \leq\left(\frac{2 N}{a}+1\right) e^{-N}+\frac{2 e^{-N}}{1-e^{-a}}=N e^{-N}\left(\frac{2}{a}+\frac{1}{N}\left(\frac{3-e^{-a}}{1-e^{-a}}\right)\right) .
\end{aligned}
$$


Proposition 7 shows that $\left\{E_{m b(N)} T_{n a} \varphi_{N}\right\}_{m, n \in \mathbb{Z}}$ is a frame with canonical dual frame $\left\{E_{m b(N)} T_{n a} \tilde{\varphi}_{N}\right\}_{m, n \in \mathbb{Z}}$ and lower frame bound

$$
A\left(\varphi_{N}\right)=\frac{\left|\varphi_{N}\left(\frac{a}{2}\right)\right|^{2}}{b(N)}=\frac{\left(g\left(\frac{a}{2}\right)-g(N)\right)^{2}}{b(N)}=\frac{e^{-a}\left(1-e^{-N+\frac{a}{2}}\right)^{2}}{b(N)}
$$

as in Corollary 11 the result now follows from Theorem 9 and Remark 10.

For (2), we use the estimates

$$
\begin{aligned}
Q_{N}(b(N)) & =(\lfloor 2 b(N) N\rfloor+1) \varphi(N)+2 \sum_{k=0}^{\infty} \varphi\left(N+\frac{k}{b(N)}\right) \\
& \leq 2 \varphi(N)+2 \sum_{k=0}^{\infty} \varphi(N+2 N k) \\
& \leq 2 e^{-N^{2}}+2 \sum_{k=0}^{\infty} e^{-N^{2}-4 N^{2} k}=e^{-N^{2}}\left(2+\frac{2}{1-e^{-4 N^{2}}}\right)
\end{aligned}
$$

and

$$
\begin{aligned}
Q_{N}\left(\frac{1}{a}\right) & =\left(\left\lfloor\frac{2 N}{a}\right\rfloor+1\right) \varphi(N)+2 \sum_{k=0}^{\infty} \varphi(N+a k) \\
& \leq\left(\frac{2 N}{a}+1\right) e^{-N^{2}}+2 \sum_{k=0}^{\infty} e^{-N^{2}-2 a N k}=N e^{-N^{2}}\left(\frac{2}{a}+\frac{1}{N}\left(\frac{3-e^{-2 N a}}{1-e^{-2 N a}}\right)\right) .
\end{aligned}
$$

Again $\left\{E_{m b(N)} T_{n a} \varphi_{N}\right\}_{m, n \in \mathbb{Z}}$ is a frame with canonical dual frame $\left\{E_{m b(N)} T_{n a} \tilde{\varphi}_{N}\right\}_{m, n \in \mathbb{Z}}$ and lower frame bound

$$
A\left(\varphi_{N}\right)=\frac{\left|\varphi_{N}\left(\frac{a}{2}\right)\right|^{2}}{b(N)}=\frac{\left(g\left(\frac{a}{2}\right)-g(N)\right)^{2}}{b(N)}=\frac{e^{-\frac{a^{2}}{2}}\left(1-e^{-N^{2}+\frac{a^{2}}{4}}\right)^{2}}{b(N)},
$$

and the conclusion follows as before.

Remark 13 The 2 standard functions in Corollary 12 are both convex on $\left[N, \infty\left[\right.\right.$ when $N \geq 1$, and so the bound $\frac{1}{b} Q_{N}(b) Q_{N}\left(\frac{1}{a}\right)$ for $B\left(q_{N}, a, b\right)$ that is used in the proof of Theorem 9 can be replaced by a sharper bound according to Proposition 5. This yields sharper bounds in Corollary 12 (1) and (2).

Acknowledgment The authors would like to thank the reviewers for several useful suggestions, which improved the presentation of the results. 


\section{References}

1. Bui, H., Laugesen, R.: Frequency-Scale Frames and the Solution of the Mexican Hat Problem. Constr. Approx. 33, 163-189 (2011)

2. Christensen, O.: An introduction to frames and Riesz bases. Second expanded edition. Birkhäuser, Boston, 2016.

3. Christensen, O., Kim, H.O., Kim, R.Y.: B-spline approximations of the Gaussian, their Gabor frame properties, and approximately dual frames. J. Fourier Anal. Appl. accepted for publication. doi:10.1007/s00041-017-9557-3

4. Christensen, O., Laugesen, R.: Approximately dual frames in Hilbert spaces and applications to Gabor frames. Sampl. Theory Signal Image Process. 9, 77-90 (2011)

5. Dörfler, M., Matusiak, E.: Nonstationary Gabor frames - approximately dual frame and reconstruction errors. Adv. Comput. Math. 41, 293-316 (2015)

6. Feichtinger, H. G., Grybos, A., Onchis, D. M.: Approximate dual Gabor atoms via the adjoint lattice method. Adv. Comput. Math. 40, 651-665 (2014)

7. Feichtinger, H. G., Onchis, D. M., Wiesmeyr, C.: Construction of approximate dual wavelet frames. Adv. Comput. Math. 40, 273-282 (2014)

8. Janssen, A.J.E.M.: Some Weyl-Heisenberg frame bound calculations. Indag. Math. 7, 165-183 (1996)

9. Janssen, A.J.E.M.: On generating tight Gabor frames at critical density. J. Fourier Anal. Appl. 9, 175-214 (2003) 